\title{
DISTRIBUTION AUTOMATION ON LV AND MV USING DISTRIBUTED INTELLIGENCE
}

\author{
Francisco MELO \\ EDP Distribuição - Portugal \\ francisco.melo@edp.pt \\ Nuno SILVA \\ EFACEC - Portugal \\ nuno.silva@efacec.com
}

\author{
Carlos CÂNDIDO \\ EDP Distribuição - Portugal \\ carlos.candido@edp.pt \\ Filipe CAMPOS \\ EFACEC - Portugal \\ fcampos@efacec.com
}

\author{
Carlos FORTUNATO \\ EDP Distribuição - Portugal \\ carlos.fortunato@edp.pt \\ Paulo REIS \\ Silver Spring Networks - Portugal \\ preis@ silverspringnet.com
}

\begin{abstract}
Nowadays, the electrical energy distribution network is considered to be a critical infrastructure in industrially developed societies. Its protection regarding safety and security threats is increasing awareness and concern. The fact that this infrastructure is geographically spread across large areas brings difficult technological challenges for fault management (real-time prevention, detection, precise location and isolation of anomalies). The distribution automation and fault location in the distribution network is a key task for any operator.

Distribution Automation (DA) and electric network monitoring and sensing can reduce outage and repair times, optimise voltage profiles and improve asset management. Advanced DA processes real-time information from sensors, smart meters, Distributed Generation (DG) and network topology for fault location, automatic reconfiguration of feeders, voltage and reactive power optimisation. This paper addresses this subject and describes the technology and functionalities being implemented on a DA pilot project promoted by EDP Distribuição (EDP Group), Portugal and under InovGrid initiative together with EFACEC and Silver Spring Networks (SSN).
\end{abstract}

\section{INTRODUCTION}

Since the early 90 s with the deployment of automation, namely reclosers, electrical distribution companies have managed to improve System Average Interruption Duration Index (SAIDI) indicators. Remote operation of switches and reclosers became available to the operator.

The geneses of these equipments are still working in many electrical grids. It is possible to have feedback from the equipments, to perform local and remote manoeuvres and detect and isolate a fault.

However, there is a limitation for deploying these equipments over MV lines, namely over the same feeder. This kind of deployment obliges coordination between reclosers or even with the main feeder breaker at the primary substation (feeder exit) in order to identify the fault. Therefore, a large number of reconnections can occur, leading to System Average Interruption Frequency Index (SAIFI) degradation and, also, a degradation of quality of service is noticed by electrical clients.

Nowadays, a new generation of reclosers detects the passage of a short circuit current and isolate the fault. This has a direct benefit on SAIFI indicators and therefore the quality of service perception by clients improves. However these new generation reclosers are costly when compared to simpler and less intelligent versions.

The solution for self-healing grids (MV) presented in this paper is based on the Distribution Automation concept with distributed intelligence, that is, information is aggregated at the primary substation and a decision of reconfiguration is also calculated at the same level. With this kind of solution, the equipments deployed along the network do not need to be provided with additional functionalities, they just need to report data/ status and act according to the orders received from the Fault Detection Isolation and Restoration (FDIR) system. Therefore, several benefits on reliability performance can be achieved.

Also, the goal of this project goes beyond Medium Voltage (MV) and includes the deployment of automated features in Low Voltage (LV) grid. With "last gasp" functionality from smart meters it is possible to identify an outage in the LV grid and identify the zone where it has occurred. This is an opportunity to implement proactive detection strategies in LV grid outages.

The solution proposed in this paper will be validated on a pilot project which will assess the positive impact of the integration of the presented technologies on network operation.

\section{REFERENCE ARCHITECTURE - INOVGRID}

The overall solution (Figure 1) is comprised of several interconnected components which allow addressing the goals set for the system contributing for an Active Network Management. The main components of this solution are: i) Smart Meters, ii) Distribution Transformer Controller (DTC), iii) Smart Substation Controller (SSC), iv) Remotely operated switches and Fault Current Indicators (FCI), v) Networks / Communications and vi) Information Systems. 


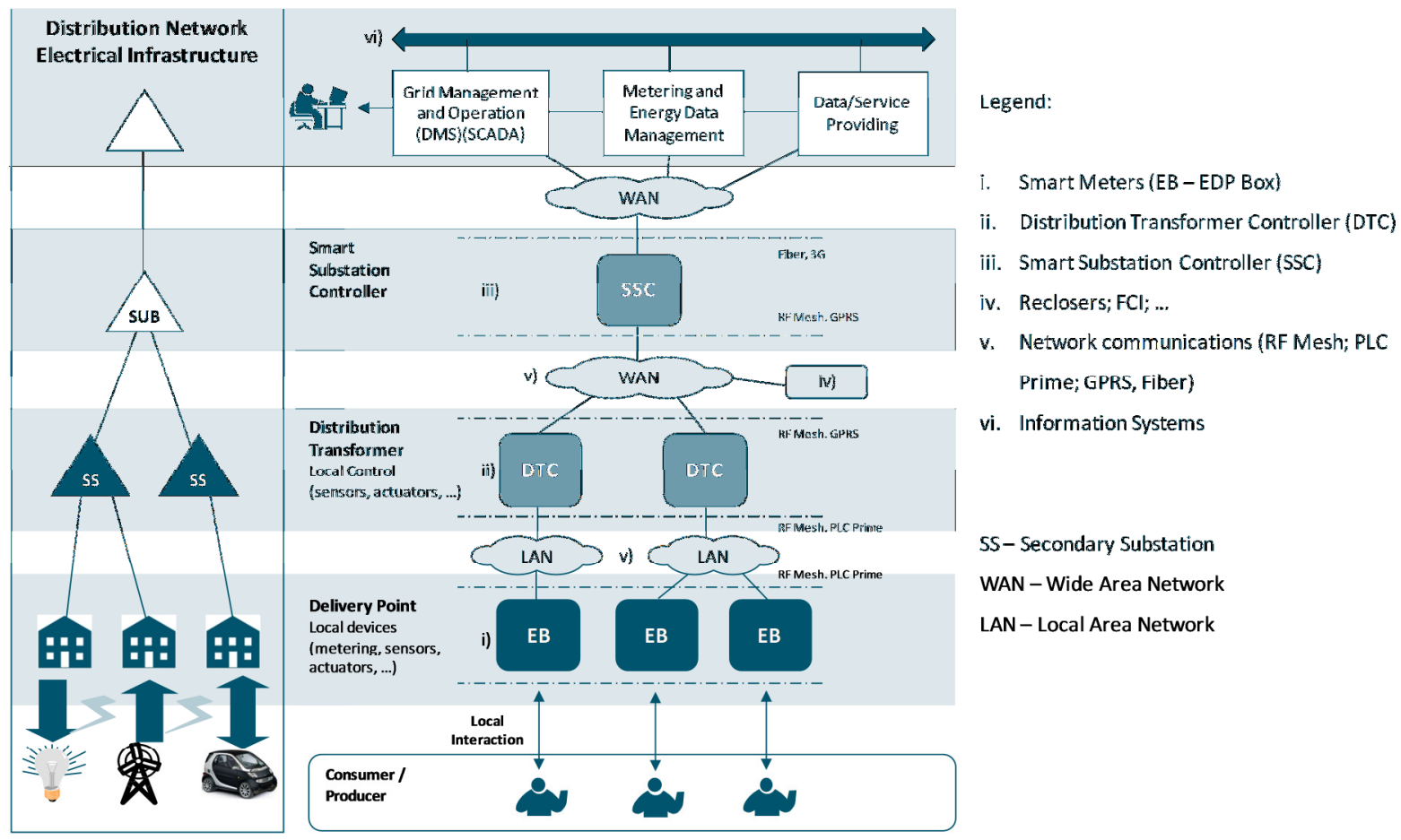

Figure 1 - Illustration of technical reference architecture of the DA project

The DTC clusters the smart meters and microgeneration on the LV network and allows multiple communication interfaces to these devices. It is also able to automatically configure and process real-time operational data and alarms as well as commercial and client information.

This reference architecture is implemented in the Portuguese Smart Grid project - InovGrid. The InovGrid project was selected among 220 smart grid projects by Joint Research Centre (JRC) of the European Commission (EC) and Eurelectric as the single case study for testing and validation of Business Case assessment methodology (EPRI).

\section{Smart Substation}

The main goal of the Smart Substation Controller (SSC) is to manage the various controllable devices on the MV network and the DTCs installed at secondary substations. The SSC concept represents an evolution on network operation, when interacting with devices on the MV network, it provides a higher number of control variables. The SSC has topology processing capabilities, being able to run load flows and execute Fault Detection, Isolation and Restoration (FDIR) using remotely operated switches and reclosers, without the need of central SCADA/DMS. That is, the self-healing capability requires no human intervention with positive impact on the reduction of outage time. Therefore, optimization of network operation is assured through algorithms that can minimize outage times, reduce technical losses and increase the penetration of DG in an efficient and coordinated manner.

\section{SELF-HEALING - MV GRID}

As utilities face increasing demand for availability, high security, cost reduction and improved quality of service, they need to cope with a pragmatic use of power assets, renewable DG and Smart Grid advanced applications.

A centralized dispatcher monitored Distribution Management System (DMS) plays an important role within this scope. These types of solutions are already in place in some distribution network operators with high levels of automation. Many primary distribution systems are designed and constructed as meshed networks, but are operated as radial feeder systems with normally-open tie switches. These tie-switches can help transferring unfaulted, but out-of-service load to neighbouring feeders to reduce the total load that has to be cut off over a prolonged period after a fault.

Whenever there is a fault in an electrical distribution system, in order to ensure minimal reduction of system reliability, the unfaulted de-energized areas should be supplied with power as soon as possible. Although repairing the fault may take a while, it is possible to quickly restore power to areas cut off by the fault or the consequent protections tripping if they can be temporarily connected to neighbouring feeders carrying the extra load.

Typically, this process is performed by an operator. When a fault occurs on a MV line, the protection switch at the primary substation is actuated and reconnection attempts are made to mislead transitory defects (e.g.: tree that touched an overhead line). If it is a permanent fault, the network operator can operate the remotely controlled switches in order to minimize the number of customers affected by the outage. Additionally, the placement of Fault Current Indicators (FCI) with communication capability allows the enrichment of the quality of information reflecting a more accurate location of the 
fault.

Automatic feeder sectionalizing and restoration is a core application for DA. In their most basic form, these systems detect a fault, determine its location, and open the nearest available switches or fault interrupters during a tripped state of the fault-clearing recloser or breaker, isolating the faulted segment from the rest of the feeder. Automatic Fault Detection, Isolation and Restoration (FDIR) algorithms carry out complex network selfhealing.

\section{Self-healing main goals}

- Supply maximum load affected by the fault

- Take the shortest time period to restore the load

- Minimize the number of switching operations

- Keep the network capacity within its operating limits

Constraints and issues

- Cables/lines loading and transformer capacity

- Voltage constraints

- Time-of-day loads (where the considered load could be the maximum load for a period after the fault occurs)

- Loads classification (priorities)

- Load shedding

- Number of switching operations

- Minimizing losses

- Sequence of switching operations

- Fault current sensitivity in the event of a subsequent fault to the extended feeder

As mentioned before, there are examples of implementation of these self-healing strategies in some distribution network operators. However, these mechanisms can also be implemented using substationcentric solutions, based on micro-DMS running on substations (SSC) with real time knowledge of their own downstream network topology, providing optimum fully automatic local switching plans.

The implementation of self-healing strategies supported by distributed intelligence at the primary substation level, taking advantage of all the information gathered from lower levels (smart meters, DTC and switching devices) is one of the goals included in this pilot project. Based on a model driven intelligence sited at the HV/MV substation, coordinating feeders and neighbouring substations, the decision process is made at the substation. It grants interoperability supporting different switches, reclosers and RTU vendors, assuring adaptability to any real-time network configuration, including any protection or automation plans. Though the operation area is restricted to the area where the substation-centric solution performs, covering a specific Distribution Grid Area (DGA) [2], it is able to dynamically derive complex restoration solutions involving multiple feeders, using where necessary, automatic load transfer schemes to achieve optimal restoration, besides offering flexibility for DG management. A DGA is an operational area defined by one or more substations and their feeders, with possibility of automatic reconfiguration in the event of a fault. Within this area, it is possible to restore non-supplied loads executing few switching manoeuvres to perform load transfer between feeders. In this project, a DGA is created consisting on $3 \mathrm{HV} / \mathrm{MV}$ substations with load transfer possibilities (network operated on open-loop).

The importance of automation devices placed in primary substation and MV lines results in an increase of the quality of service, reduction of interruption time, number of customers affected and energy not supplied. The information collected by sensors (e.g.: FCIs) combined with the dynamic model of the network topology and real time information about energy flows will allow the system to make a decision about network reconfiguration. For this, we need algorithms for analyzing real-time multivariable system energy.

In the Inovgrid architecture, the SSC has topology processing capabilities, being able to run load flows and execute Fault Detection, Isolation and Restoration (FDIR) using switches and reclosers, without the need of central SCADA/DMS (Figure 2). That is, the self-healing capability requires no human intervention with positive impact on the reduction of outage time. Therefore, optimization of network operation is assured through algorithms that can minimize outage times, reduce technical losses and increase the penetration of DG in an efficient and coordinated manner.

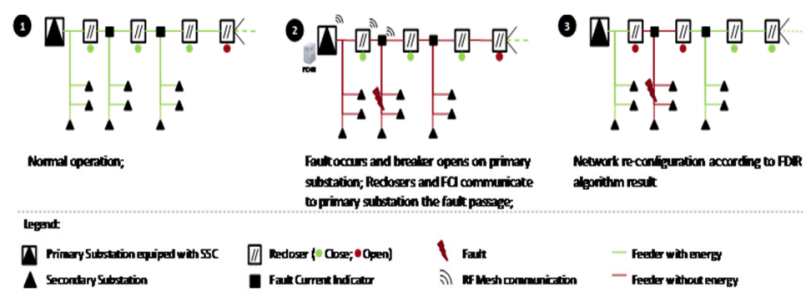

Figure 2 - Illustration of FDIR behavior after a fault on MV network

\section{COMMUNICATIONS - POWER OUTAGE}

A power outage affects communications network devices in various ways. Communication network equipment (Access Points and Relays) installed with an optional battery backup can operate through outages of at least eight hours. Electricity meters typically send a last gasp, save their state, shut down gracefully, and use battery power to maintain their real-time clocks.

An Access Point with a battery backup can remain functional during an extended loss of power. During an outage, the Communications Network Management System (CNMS) polls network devices to help determine what devices are still reachable. During an outage, the CNMS can only determine if it can reach devices. If it can reach a device, the device in question is either unaffected by the outage or is operating on battery backup itself, in which case it issues an alarm.

If it cannot reach a device, it does not necessarily mean 
the device is without power, only that it cannot be reached. There may be several reasons why a device cannot be reached, including a situation where some devices still have power but cannot be reached because intervening hops are affected by the outage. Hence the inability to reach a direct child device probably means the child device has also lost power, but inability to reach the children of child devices is ambiguous. While powered by battery backup, communications devices will be able to detect when their backup battery is nearly exhausted, send a signal to the CNMS, and gracefully shut down.

When power is restored, these devices return to full function and send the appropriate events to AMM.

\section{LAST GASP - LV GRID}

Fault detection functionality for LV networks can be achieved through an intelligent use of Advanced Metering Infrastructure (AMI). Smart Meters and LV sensors with RF Mesh communications will report alarms on-event to the DTC, such as power failures on LV feeders.

Since meters have no battery power backup, when the meter detects a low voltage event on the LV network, it will report the event to the NIC and then shutdown gracefully.

The NIC will transmit the Last Gasp message to the nearest DTC (working as access point) that will relay this message to the DTC that is supplying that meter (Figure 3 ). The corresponding DTC will then report the alarm to the central systems.

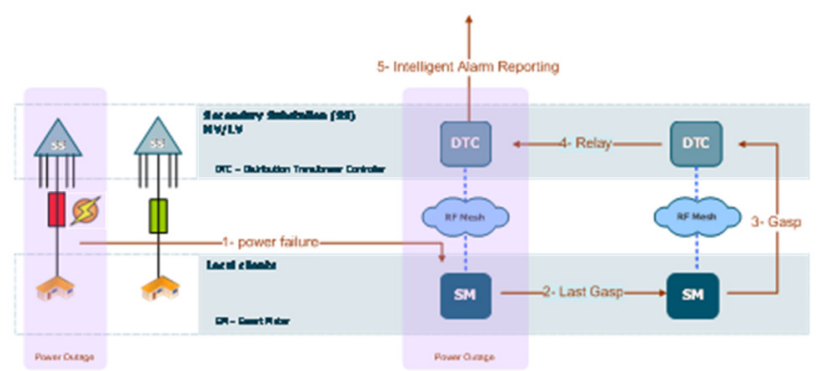

Figure 3 - LV fault detection using Last Gasp

Due to the criticality of electric distribution networks nowadays, the goal of this project is to determine the positive impact of this conceptual design of the explained technology integration on network operations.

\section{CONCLUSIONS}

EDP Distribuição and its stakeholders expectations involve achieving higher standards on quality of service, thus providing focus on the permanent development in distribution automation solutions.

Distribution Grids need to become smarter so that they support a decentralized control and monitoring structure across the medium and low voltage network, the automation of grid operations, an automatic detection of fault conditions and initiation of restoration actions and dynamic adaptation mechanisms for active management of grid constraints and voltages. This pilot will deploy a decentralized control and monitoring structure across the medium and low voltage network. The pilot project will allow verifying if the conceptual design here described positively translates into network operations providing support to the decision for a potential subsequent roll-out.

\section{REFERENCES}

[1] R. Oliveira et al., 2009'Increasing quality of supply of EDP through optimal and strategic distribution automation design", 20 $0^{\text {th }}$ CIRED International Conference, CIRED, paper 459.()

[2] A. Bernardo et al., 2011, "Preventive assessment for combined control centre and substation-centric self-healing strategies", Proceedings 21st International Conference on Electricity Distribution, CIRED, paper 508.

[3] A. Bernardo et al., 2012, "DG behaviour and protection schemes in case of disturbances within the concept of distribution grid area", Proceedings CIRED Workshop 2012, paper 356.

[3] A. Madureira et al., 2009, "The new role of substations in distribution network management", Proceedings 21st International Conference on Electricity Distribution, CIRED, paper 863.

[4] Engineering Recommendation P2/6, 2005, "Security of Supply", Energy Networks Association, Engineering Directorate, UK.

[5] I. Waseem et al., 2009, "Reliability benefits of distributed generation as a backup source", Proceedings of PES General Meeting, IEEE PES'09, Calgary, USA. 